\title{
Métrologie des radionucléides présents à l'état de traces dans l'environnement. Normalisation des procédures et traçabilité des essais
}

\author{
D. Calmet
}

Institut de Protection et de Sûreté Nucléaire, Département de Protection de l'Environnement, Service d'Études et de Recherches sur les transferts dans l'Environnement et Laboratoire de mesure de la radioactivité de l'environnement, Bâtiment 501, Bois des Rames, F-91400 Orsay Cedex, France

\begin{abstract}
Widespread concern over radioactive substances in the environment regularly requires environmental and public health impact assessments. The credibility of an assessment will depend on the quality and reliability on measurement results that often are of paramount significance in the environmental domaine. Those man made radionuclides present in the various environmental components of the French territory are however found at trace, even ultra-trace levels. This article gives an overview of standardization work and required reference materials and rules for measuring radionuclides in environmental matrices as well as the international and national systems to manage standardization and traceability. Some achievements as well as the many difficulties that the metrologist must overcome when using nuclear techniques to measure trace quantities of radionuclides are presented.
\end{abstract}

\section{Introduction}

La préexistence et les ajouts, autorisés ou accidentels, de substances radioactives dans l'environnement justifient les demandes d'expertises d'impacts sur l'environnement (radioécologie) et les évaluations d'impacts sur les populations humaines (radioprotection). Ces questions émanent tant des instances officielles internationales, nationales ou régionales que des exploitants d'installations nucléaires et également des médias et des associations publiques. Dans ce contexte, même si les niveaux de radioactivité attendus dans l'environnement sont faibles, des résultats de mesurages sont nécessaires pour établir l'impact des pratiques ou des accidents nucléaires en cause [1]. Les résultats de ces mesurages seront utilisés pour établir des états radiologiques ponctuels, des chronologies sur le long terme ou pour expliciter et quantifier les mécanismes de transfert des radionucléides entre les différents constituants de l'environnement. Ils seront éventuellement confrontés aux résultats des calculs, obtenus par des simulations mathématiques, afin de valider les évaluations théoriques d'impacts et de risques. La crédibilité des expertises dans le domaine nucléaire repose donc, en partie, sur la qualité et la fiabilité des résultats de mesurages, car comme l'indiquait plus généralement un rapport récent de l'Académie des Sciences, "les questions de mesure occupent une place centrale dans le domaine de l'environnement. » [2].

Les radionucléides peuvent être identifiés, puis quantifiés, suivant deux approches. L'une qui est généralement considérée comme plus sensible pour les éléments ayant des périodes radioactives supérieures à 1000 ans, repose sur l'identification des masses atomiques par spectrométrie de masse. L'autre approche consiste à détecter les émissions radioactives des radionucléides lors de leur décroissance et est bien adaptée aux isotopes ayant des périodes inférieures à 1000 ans. Dans ce dernier cas, la spectrométrie gamma germanium haute résolution, la spectrométrie alpha et la scintillation liquide à bas mouvements propres ont une place de choix [3]. Aujourd'hui, le niveau de développement de ces techniques a dépassé le stade de la maturité [4] car les mécanismes en jeu sont bien compris, les principes établis et des équipements de mesurage performants sont disponibles. En radioécologie et en radioprotection, ce sont des techniques de mesurage de base pour mesurer un grand nombre de radionucléides émetteurs alpha, bêta et gamma susceptibles d'être présents dans l'environnement, qu'ils soient d'origine naturelle ou artificielle, bien que les techniques ICP-MS soient en plein développement méthodologique actuellement. Elles sont utilisées de plus en plus largement et ce dans des domaines d'applications variés. Ce degré de développement se traduit, entre autres, par l'existence de documents normatifs décrivant les protocoles de mesurage $[5-7$, etc. $]$.

Le mesurage par une technique nucléaire d'un radionucléide, présent dans un échantillon de l'environnement, consiste à déterminer la grandeur « activité », dont l'unité est le Becquerel. Les méthodologies et les différentes techniques de mesurage des nucléides ont été adaptées aux 
matrices environnementales : gaz, aérosols, eaux naturelles, sol, sédiments, organes de végétaux ou d'animaux considérés comme bioindicateurs. Elles doivent également être ajustées aux questions posées lors de l'établissement de l'état radiologique de l'environnement, puis lors de l'évaluation de l'impact des nucléides radioactifs sur les écosystèmes et du risque pour les populations humaines. Les méthodologies et les installations de mesurages doivent également être adaptées aux développements et modifications des pratiques liées aux applications nucléaires qui conduisent à la baisse progressive des rejets de radionucléides dans l'environnement. Ainsi, entre mai 1986, date de l'accident de Tchernobyl, et aujourd'hui, l'activité des radionucléides artificiels, comme le césium 137, a décru dans l'atmosphère d'un facteur $10^{6}$ [8]. Ces activités, représentatives de celles des différentes composantes aquatiques et terrestres de la plus grande partie du territoire français, relèvent du mesurage des radionucléides dans le domaine des traces ${ }^{1}$, voire des ultra-traces. Cependant, dans tous les cas, les méthodologies doivent être conformes aux exigences des normes ISO pour les laboratoires d'essai [9] afin de garantir la fiabilité des résultats de mesurage nécessaires à la crédibilité des études et expertises ainsi que du contrôle réglementaire.

Depuis la découverte des rayons $X$ et de la radioactivité à la fin du siècle dernier, la métrologie des rayonnements ionisants s'est développée parallèlement aux applications médicales, industrielles et militaires. Un ensemble de grandeurs et d'unités spécifiques de la métrologie des rayonnements ionisants a été défini et les moyens nécessaires à la réalisation des étalons et des raccordements métrologiques nécessaires aux différentes applications ont été mis en place dans la plupart des pays industrialisés. L'internationalisation des activités et pratiques nécessitant des mesurages, en particulier celles touchant le nucléaire et l'environnement, conduit à l'étude des contaminations transfrontières industrielles, chroniques ou accidentelles. Il faut donc que les résultats établis dans un pays soient également admis comme fiables dans les pays potentiellement concernés par une contamination régionale. Cela exige que les résultats d'activités obtenus par différents laboratoires d'essais puissent être reproduits et comparés.

Cet article présente brièvement comment s'insèrent les travaux de normalisation et les règles de raccordement nécessaires en métrologie des radionucléides présents dans les matrices environnementales au sein des organisations internationales et nationales de normalisation et de traçabilité. Les acquis, mais aussi certaines des difficultés auxquelles le métrologiste utilisant les techniques nucléaires doit faire face dans le domaine des traces sont exposées. Par contre cet article ne traite pas des aspects relevant de la métrologie en radiochimie, très proches de ceux de la chi-

\footnotetext{
${ }^{1}$ De nombreux analystes utilisent le terme de traces pour décrire des déterminations réalisées à des niveaux inférieurs à une partie par million (ppm), d'autres l'utilisent pour des analyses où l'analyte est à des niveaux de concentrations qui posent des difficultés techniques pour l'obtention de résultats validés.
}

mie dont elle n'est qu'une branche, et qui fait l'objet d'une présentation spécifique dans cette revue (Vialle J. et al. dans ce numéro).

\section{Contraintes associées au mesurage de radionucléides à l'état de traces}

L'activité des radionucléides artificiels présents actuellement dans la majorité des différentes composantes de l'environnement relève généralement de l'analyse des traces (Fig. 1). Ainsi des éléments pourtant considérés encore aujourd'hui comme communs dans l'environnement, tel que le césium 137, sont quantifiés à des niveaux inférieurs aux microBecquerel. $\mathrm{m}^{-3}$ dans l'air et relèvent du domaine des ultra-traces. Dans ce domaine, les problèmes rencontrés pour des échantillons de l'environnement et ce, quel que soit le type d'analyse [10], peuvent être résumés comme suit :

(i) l'activité de l'analyte est très inférieure à celles des autres constituants, entre autres naturelles, de la matrice ;

(ii) des contaminations provenant de réactifs (y compris l'eau de laboratoire), des équipements ou de l'environnement du laboratoire peuvent conduire à des résultats surestimés ;

(iii) les pertes de l'analyte à mesurer, lors des opérations de préparation radiochimiques ou autres, sont plus critiques aux faibles concentrations et peuvent conduire à être dans l'incapacité de détecter l'analyte lorsque celui-ci est présent à des activités proches de la limite de détection de la technique de mesurage ;

(iv) l'interférence des constituants de la matrice avec le système de détection peut conduire à des concentrations sous-estimées ou surestimées qui nécessitent de rajouter des

\section{Radioactivité naturelle dans l'air}

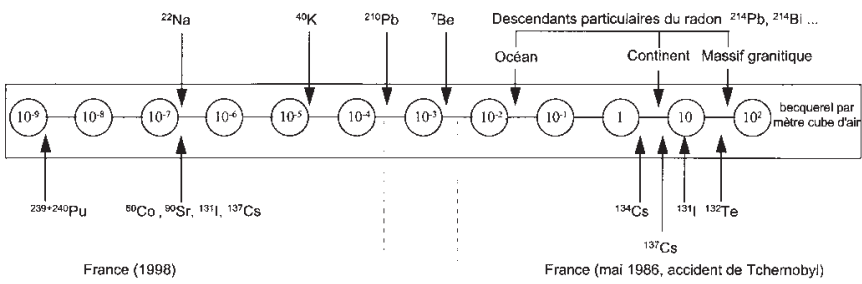

Radioactivité artificielle dans l'air

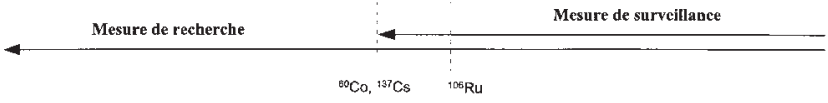

Figure 1. Échelle indicatrice des niveaux de radioactivité des aérosols atmosphériques et des domaines de mesurage respectifs d'un laboratoire de contrôle de l'environnement classique et celui impliqué dans des recherches en radioécologie. 
étapes de purification ou l'utilisation de détecteurs plus sélectifs ; et enfin

(v) la vérification de la fiabilité des méthodes de mesurage dans le domaine des traces est difficilement traçable à cause de la rareté des matériaux de référence primaires et secondaires certifiés adaptés à ces activités.

Afin de positionner les besoins métrologiques en domaine nucléaire, nous utiliserons une illustration graphique (Fig. 2), de la dégradation de la fiabilité des mesurages d'analytes en fonction de leurs concentrations dans différents types de matrices jusque dans le domaine des traces (ng g ${ }^{-1}$ ) déduite des travaux de Horwitz et al. [11]. Il apparaît que le coefficient de variation entre des résultats d'intercomparaisons obtenus par différents laboratoires est une fonction inverse des niveaux de concentrations mesurées classiquement. Or comme nous venons de le voir, pour comparaison les concentrations classiquement observées en césium 137 dans les différentes matrices de l'environnement se situent actuellement à $10^{-15}$, quasiment hors échelle pour les concentrations les plus faibles. Dans ce contexte, garantir la fiabilité des résultats obtenus est considéré comme la question majeure de l'analyse des traces de radionucléides dans les matrices environnementales.

Ces questions font l'objet de publications régulières [12-15] et conduisent parfois à la mise en cause des procédures de mesurage des radionucléides et des étalons utilisés inadaptés d'une part aux niveaux des traces d'activité et, d'autre part, aux matrices environnementales. La seule

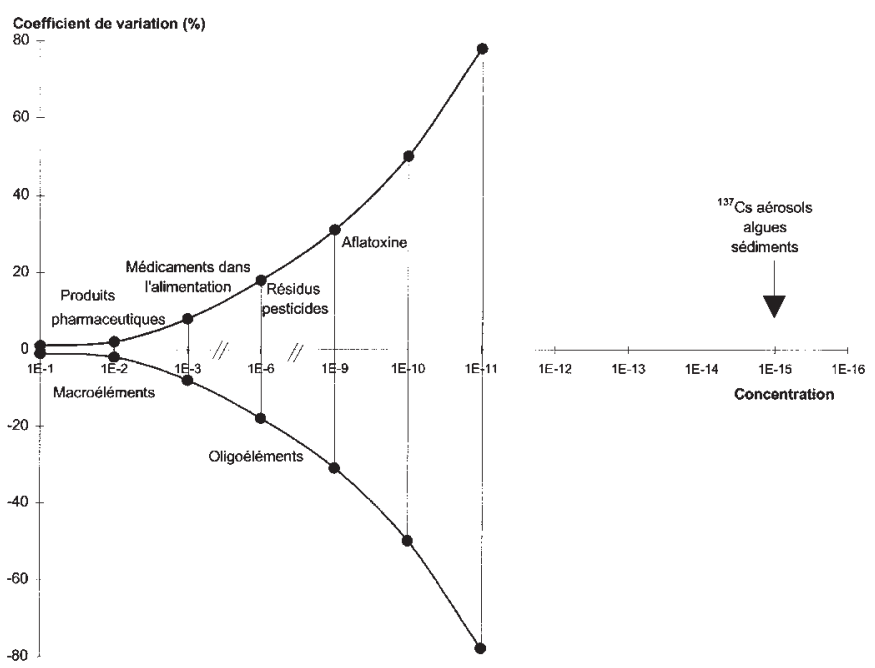

Figure 2. Évolution du coefficient de variation des résultats obtenus au cours d'essais d'aptitude interlaboratoires en fonction du niveau de concentrations des différents analytes au sein de différentes matrices. Les concentrations en masse correspondant aux niveaux d'activités en césium 137 mesurés dans différentes matrices environnementales en France à l'heure actuelle sont reportées (modifié d'après [9]). réponse possible est la mise en place d'un système d'assurance de la qualité rigoureux, en particulier, en respectant les bonnes pratiques de laboratoire [16] et les contraintes énoncées dans les principes de l'assurance de la qualité, codifiées dans les documents de la série de l'ISO 9000 et 9001 pour la certification et du guide ISO/CEI N ${ }^{\circ} 25$ [9], future norme ISO 17025, pour l'accréditation.

\section{Organisation de la normalisation dans le domaine nucléaire}

Les organisations s'occupant au niveau national français de la normalisation en métrologie, le Bureau de normalisation des équipements nucléaires (BNEN) et l'Association française de normalisation (AFNOR) et, au niveau international, le Comité européen de normalisation (CEN) et l'International standardization organisation (ISO), s'efforcent de mettre en place une pratique métrologique rigoureuse pour le mesurage de l'activité des radionucléides afin d'assurer la comparabilité des résultats obtenus dans les différents pays.

\section{Organisation au niveau international}

$\mathrm{Au}$ sein de l'ISO, c'est le comité technique 85 chargé des différents aspects de l'utilisation de l'énergie nucléaire qui regroupe, dans le sous-comité Radioprotection, les activités de normalisation qui intéressent le mesurage des radionucléides. À partir de 1998, c'est au sein du groupe de travail $\mathrm{N}^{\circ} 17$ que sont traitées plus particulièrement les questions de métrologie de la radioactivité de l'environnement (Fig. 3). Il est prévu d'y comparer les différentes normes nationales concernant le mesurage de la radioactivité dans l'environnement, émises par les pays européens, France (AFNOR), Allemagne et Pays-Bas ainsi que par les ÉtatsUnis d'Amérique (AMST) afin de les éditer sous la forme de normes ISO.

\section{Organisation en France}

$\mathrm{Au}$ niveau français, créé le 26 juin 1990 par décision du Ministère de l'industrie et de l'aménagement du territoire, le

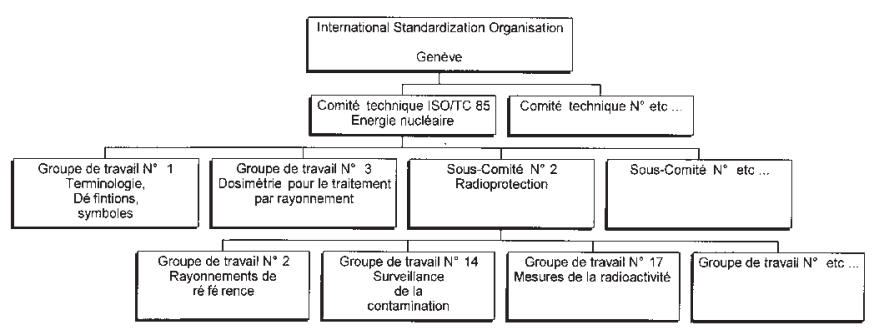

Figure 3. Organisation du mesurage de la radioactivité au sein des groupes de travail de I'International Sandardization Organisation et positionnement du groupe de travail $N^{\circ} 17$ traitant du mesurage de la radioactivité. 
Bureau de normalisation des équipements nucléaires est en charge de la gestion des commissions françaises et des groupes de travail associés, dans les domaines de la protection contre les rayonnements ionisants (M60-1), de la technologie du cycle du combustible nucléaire (M60-2) et du mesurage de la radioactivité dans l'environnement (M60-3). Cette dernière commission a été créée en 1992 à la suite des litiges au sujet de la décharge banalisée sur l'ancien site militaire du Bouchet, conduisant le Ministère de l'industrie à demander à l'AFNOR de lui proposer des travaux sur les mesurages de radioactivité dans l'environnement. L'approche normative semblait pouvoir répondre à une facette des contentieux entre organismes publics et organismes privés effectuant les mêmes mesurages de radioactivité mais obtenant des résultats en apparence significativement différents.

La commission M60-3 est structurée en quatre groupes de travail en fonction des principales matrices environnementales : air, eau, sol et bioindicateurs (Fig. 4). Elle est chargée de la normalisation des méthodes de mesurage de la radioactivité dans l'environnement, à l'exception de la radioprotection des travailleurs, du domaine médical et de la contamination. Depuis septembre 1992, les travaux du Comité technique 85 de l'ISO, portant sur les limites de détection et le mesurage du radon, ont été rattachés à la commission M60-3. Depuis sa création, les travaux de cette commission ont conduit à la publication de 17 normes AFNOR sur les méthodes de mesurages des radionucléides susceptibles d'être présents au sein des différents types d'échantillons de l'environnement.

\section{Organisation de la traçabilité dans le domaine nucléaire}

Le système métrique décimal a été institué en France par la loi du 18 germinal an III, le 7 avril 1795, et en 1875 à l'occasion de la Conférence diplomatique du Mètre, dix-sept états s'engageaient à fonder le Bureau international des poids et mesures (BIPM) à Paris, afin d'assurer le système métrique dans le monde. Le système métrique originel se transformera progressivement pour donner naissance au Système international d'unités (SI) en 1960. Le développement rapide de l'utilisation des rayonnements ionisants nécessita très rapidement une cohérence de résultats entre techniques et méthodes de mesurage et c'est très logiquement que le BIPM fut retenu pour y déposer, dès 1910, un premier étalon international pour le radium, préparé par Marie Curie, puis un second préparé par Hönigschmid en 1934.

Dans le domaine de l'analyse nucléaire, l'identification et la quantification des radionucléides nécessitent la connaissance de leurs caractéristiques physiques, telles que la période radioactive, la nature, l'énergie et l'intensité des émissions des rayonnements décrit par des schémas de désintégration. Une reconnaissance au niveau international

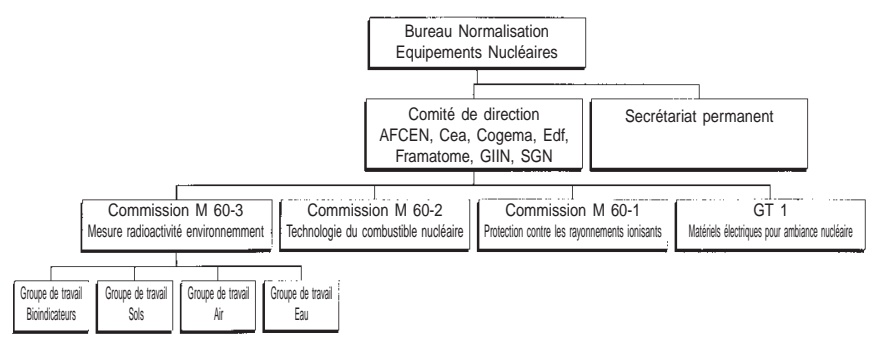

Figure 4. Organisation du Bureau de normalisation des équipements nucléaires dans lequel quatre groupes de travail y traitent spécifiquement des méthodologies de mesurage des radionucléides au sein des quatre grands types de matrices environnementales : air, eau, sols et organismes bioindicateurs.

de ces données, regroupées sous forme de table de référence des radionucléides, est rapidement apparue comme fondamentale pour asseoir le raccordement des laboratoires, primaires et autres, entre eux sur une base commune.

\section{Organisation au niveau international}

Lors du premier congrès international de radiologie en 1925 , il fut décidé de créer l'International X-Ray Unit Committee qui devint l'International Commission on Radiation Units and Measurements (ICRU), dont les travaux continuent à faire autorité dans le domaine. En 1958 fut créé le Comité consultatif pour les étalons de mesure des rayonnements ionisants (CCEMRI) au sein du Comité international des poids et mesures (CIPM) qui épaule le BIPM dans le choix

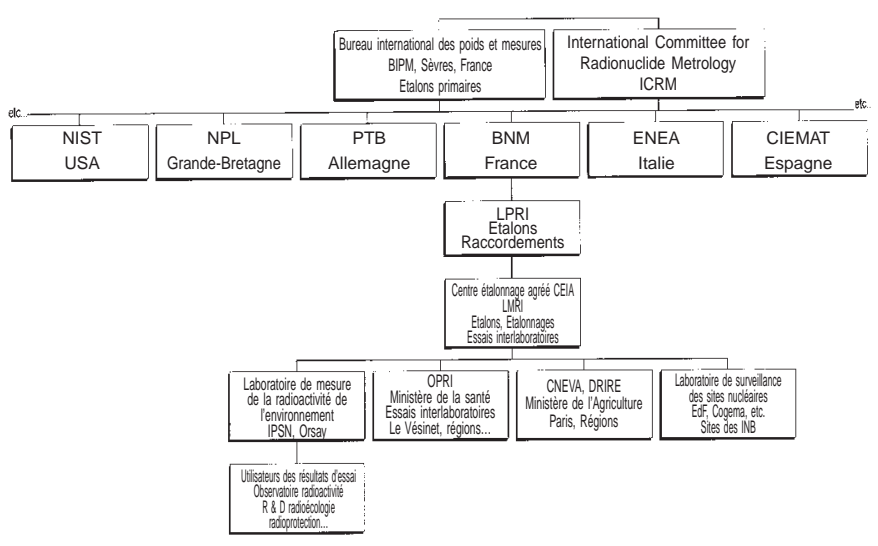

Figure 5. Organisation de la traçabilité des étalons primaires et secondaires de radionucléides naturels et artificiels (NIST : National Institute of Standards and Technology, PTB : Physikalisch-Technische Bundesansalt, NPL : National Physical Laboratory, LPRI : laboratoire primaire des rayonnements ionisants). 
de ses actions en métrologie des rayonnements ionisants (Fig. 5). Le BIPM continue en effet à jouer un rôle important en organisant des intertercomparaisons internationales de mesurage de la radioactivité. En 1975, les grandeurs et unités propres à la métrologie des rayonnements ionisants ont été référencées dans le système international d'unités. De plus, depuis 1975 également, le Système international de référence (SIR) permet aux laboratoires primaires nationaux de comparer leurs étalons de mesurage des radionucléides émetteurs gamma. Les données de base, schéma de désintégration, période radioactive, rapports d'embranchements, énergies et probabilités d'émission et de transition pour tous les rayonnements, coefficients de conversion interne et probabilités de capture électronique, regroupées sous le terme de table des radionucléides sont mises à jour régulièrement.

Au niveau européen, il faut mentionner deux organisations chargées de la coordination des actions en métrologie, à savoir le western Metrology Club (EUROMET) et l'European Cooperation for Accreditation of Laboratories (EAL).

\section{Organisation au niveau français}

En France, le Bureau national de métrologie (BNM) a été créé en 1969 avec pour mission d'animer et de coordonner les actions entreprises par les différents départements ministériels dans le domaine de la métrologie. Le BNM, par arrêté interministériel du 22 décembre 1994 a été constitué en groupement d'intérêt public pour préparer et mettre en œuvre la politique nationale de métrologie. L'une de ses missions principales est d'assurer la cohérence nationale et internationale des mesurages effectués dans les entreprises. Pour ce faire, le BNM est constitué de cinq laboratoires nationaux de métrologie qui réalisent (i) des activités de recherche fondamentale en physique et en chimie qui conduisent à de nouvelles définitions d'unités basées sur des phénomènes fondamentaux, (ii) des travaux d'amélioration et de maintien des références nationales actuelles, et (iii) le raccordement aux étalons nationaux des étalons de référence des entreprises et organismes techniques avec les meilleures incertitudes possibles.

Succédant au Laboratoire de Mesure des Radioéléments (LMR) qui fournissait des étalons de radionucléides depuis 1960, c'est le Laboratoire Primaire des Rayonnements Ionisants (BNM-LPRI) qui a été désigné comme laboratoire primaire pour le mesurage des rayonnements ionisants [17]. La production des étalons secondaires et des services associés aux besoins en métrologie a été assurée jusqu'en 1998 par le Laboratoire de Mesure des Rayonnements Ionisants (LMRI) qui est l'un des deux centres d'étalonnage agréés (CEtA). En collaboration avec le Comité Français d'Accréditation (COFRAC), les activités de ces laboratoires s'inscrivent dans la coordination des actions entreprises au sein du système des « chaînes d'étalonnage BNM-COFRAC ». Le raccordement porte sur la fourniture de sources étalons sous forme gazeuse, liquide et solide pour plus de 70 radionucléides. Le LPRI pilote également deux réseaux de laboratoires de mesurage de la radioactivité raccordés depuis maintenant plus de dix ans.

\section{Matériaux de référence}

Le mesurage des rayonnements ionisants repose, comme toute métrologie, sur la possibilité d'établir un raccordement entre la grandeur à mesurer sur un échantillon et une référence reconnue de même nature (matériau de référence primaire) et d'obtenir une évaluation de l'incertitude associée. En d'autres termes, il s'agit de réaliser la traçabilité des résultats d'essai obtenus au laboratoire en mesurant régulièrement des matériaux de références qui sont généralement détenus et certifiés par le LPRI. Les matériaux de référence primaires doivent évidemment être homogènes, stables chimiquement dans le temps et répondre à différentes contraintes variables suivant les radionucléides à mesurer. Par exemple, pour le mesurage des radionucléides émetteurs gamma par spectrométrie gamma germanium haute résolution, ils doivent couvrir l'ensemble de la gamme d'énergie $(1 \mathrm{keV}$ à $3 \mathrm{MeV}$ ), être de faible activité (de l'ordre de 3700 $\mathrm{Bq}$ ), pour obtenir une perte de temps de comptage due à la saturation de l'électronique inférieure à $1 \%$ avec un détecteur de $60 \%$ d'efficacité relative. Ces étalons doivent, en outre, couvrir une gamme de densités caractéristique de celles d'un grand nombre d'échantillons environnementaux $(0,3$ à 1,7$)$. Ces contraintes ont justifié un programme européen coordonné par EUROMET sur la mise au point de matrices marquées, artificielles et de différentes densités nécessaires aux laboratoires de contrôle des niveaux de la radioactivité de l'environnement.

\section{Matériaux de référence primaires au niveau des traces}

De nombreux étalons nationaux (français, européens et américains), sont disponibles sur catalogue sous la forme d'une solution stabilisée à l'aide d'un entraîneur stable, pour des niveaux supérieurs aux niveaux habituellement rencontrés dans des échantillons de l'environnement, par exemple quelques dizaines de $\mathrm{kBq} \mathrm{kg}^{-1}$ pour les étalons de spectrométrie gamma. Par contre, les matériaux de référence primaire (MRP) nécessaires à la calibration des installations de mesurage dédiées au mesurage des traces et ultra-traces ne sont pas élaborés sur le territoire national. Les laboratoires de mesurage réalisent alors souvent eux-mêmes des étalons secondaires dilués, non certifiés bien évidemment, à des niveaux proches de ceux qu'ils sont susceptibles de rencontrer dans les échantillons soumis à essais. Une autre solution est d'utiliser les services de sociétés privées, sous-traitant du LMRI, comme c'est le cas pour les étalons des radionucléides émetteurs gamma. Ainsi, c'est une société américaine, Analytics, qui fournit d'ores et déjà au LMRI qui les revend, les étalons aux activités des traces $(<\mathrm{kBq}$ $\mathrm{kg}^{-1}$ ) dans des matrices représentatives de l'environnement. Cette exigence de qualité et de rigueur génère un surcoût 
significatif, d'un facteur 3 par rapport aux étalons classiques qui sont fournis par le LMRI.

\section{Matériaux de référence "secondaires》 non certifiés}

Aujourd'hui, les seuls matériaux de référence secondaires (MRS) environnementaux disponibles sont ceux préparés et distribués par l'Agence internationale de l'énergie atomique (AIEA) à travers un programme intitulé Analytical Quality Control Services (AQCS). Ces échantillons de MRS sont également distribués par la société privée Promochem [18]. Ces matériaux permettent de comparer et d'évaluer les performances des laboratoires qui réalisent les mêmes types d'analyses pour des matrices de l'environnement [13]. Ces échantillons, issus d'environnements particuliers, ont été qualifiés par des exercices d'intercomparaisons internationaux (Fig. 6) mais ne peuvent généralement pas être certifiés car ils n'ont pas été élaborés à partir de matériaux de référence primaires et directement reliés à des laboratoires primaires. Aujourd'hui, dans le domaine du mesurage de la radioactivité au niveau des traces il n'existe aucun matériau de référence certifié. Les valeurs données sont soit des valeurs qualifiées de « recommandées », valeurs obtenues par consensus à la suite de comparaisons interlaboratoires, soit des valeurs dites « informatives », valeurs obtenues par consensus à la suite de comparaisons interlaboratoires mais qui ne répondent pas aux critères de qualité nécessaires pour être recommandées [19].

Le mode d'obtention de ces valeurs, recommandées ou informatives, conduit parfois à des biais. Ainsi l'analyse statistique des résultats obtenus dans des essais d'intercomparaisons AIEA portant sur l'américium 241 à l'état de traces et mesuré par deux techniques de mesurage différentes, l'une après radiochimie par spectrométrie alpha et l'autre directement par spectrométrie gamma, fait apparaître des valeurs moyennes différentes pour chaque technique. Les valeurs recommandées ou informatives intégrant l'ensemble des données se situent donc entre les valeurs moyennes associées aux deux techniques. De plus, de tels essais sont lourds à organiser, le nombre de laboratoires participants est parfois limité et les délais de publication des résultats finaux atteignent habituellement plusieurs années. L'AIEA de plus n'a pas organisé de nouvelle intercomparaison depuis 1996, et l'une des dernières s'est révélée biaisée par un défaut d'homogénéité entre les échantillons aliquotes distribués aux laboratoires participants. Ces problèmes montrent, s'il en était encore besoin, les difficultés auxquelles se heurte le métrologiste dans le domaine nucléaire et n'enlève rien à la qualité et à l'intérêt des travaux réalisés par l'AIEA. En effet, l'existence de ces exercices est fondamentale puisque, outre qu'ils débouchent sur la qualification de nouveaux MRS, la participation aux intercomparaisons est exigée pour l'accréditation des laboratoires d'essai.

\section{Matériaux qualifiés de « blancs 》}

Les radionucléides, en particulier ceux d'origine naturelle, sont présents communément dans tous les matériaux envi-

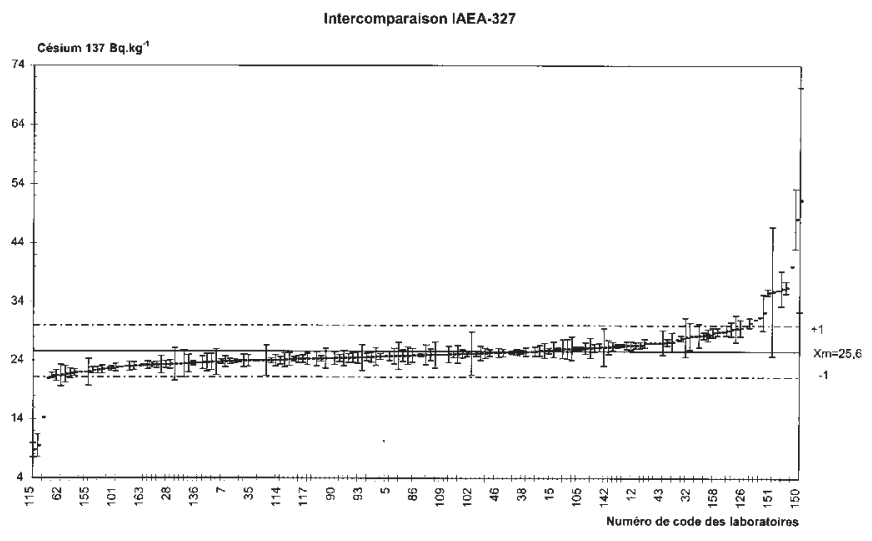

Figure 6. Résultats de césium 137 obtenus par 170 laboratoires ayant participé à l'intercomparaison AIEA-327 de type B sur des échantillons de l'environnement.

ronnementaux à différents niveaux de concentrations. Leur présence peut alors interférer lors de la radiochimie préparatoire avec les radionucléides à mesurer ayant des caractéristiques chimiques identiques ou lors de leur détection avec les radionucléides ayant des caractéristiques physiques proches. Ces difficultés sont généralement contournées pour définir un matériel dit de "blanc» en utilisant des échantillons d'eau ultra-pure ou composés de matrices synthétiques de types résines. Dans le cas particulier du mesurage d'éléments présents à la fois naturellement et également générés par les activités humaines, des objets particuliers, protégés des contaminations anthropogéniques les plus récentes sont recherchés. Ainsi, dans le cas du tritium, mesuré classiquement par scintillation liquide, ce sont des eaux de nappes phréatiques fossiles très faiblement alimentées par les eaux de surface qui constituent des échantillons de blanc [20]. En règle générale, chaque laboratoire définit des échantillons de blancs qui lui sont spécifiques, en fonction des matrices analysées et des activités à mesurer, et qui ne peuvent donc être référencés officiellement à une échelle nationale.

\section{Test d'aptitude professionnelle, certification et accréditation}

\section{Test d'aptitude professionnelle}

Les essais d'aptitude professionnelle, qui font l'objet d'intercomparaisons intra et interlaboratoires, ont pour objectif (i) de contrôler la performance d'ensemble du laboratoire d'essai, (ii) d'établir l'efficacité de la méthode d'essai, (iii) de déterminer avec un degré donné de précision, une ou plusieurs caractéristiques d'un matériau ou d'un produit, et enfin (iv) de contrôler les performances individuelles du personnel du laboratoire. 
Les modalités de réalisation des essais d'aptitude varient selon la nature du matériau ou du produit soumis à l'essai, la méthode d'essai à mettre en œuvre et le nombre de laboratoires participants. Elles ont cependant en commun le fait que des résultats d'essais obtenus par un laboratoire donné sont comparés avec ceux obtenus par un ou plusieurs autres laboratoires d'essais. Dans certains cas, l'un des laboratoires participants peut avoir une fonction de contrôle, comme c'est le cas en France avec l'Office de Protection contre les Rayonnements Ionisants (OPRI).

Différents essais d'intercomparaison et d'aptitude sont régulièrement organisés au niveau national (OPRI, CNEVA) et international (AIEA, OMS, NIST) afin de comparer et d'évaluer les performances des laboratoires (Tab. 1). Des essais d'intercomparaison sont régulièrement initiés entre deux ou trois laboratoires européens ou par les clients (EDF, Cogema) de ces laboratoires.

Les essais d'aptitude professionnelle coïncident avec les essais d'intercomparaison uniquement pour le premier objectif cité au début de ce paragraphe. La plupart des organismes qui évaluent la compétence technique des laboratoires d'essais font de la participation satisfaisante aux essais d'aptitude une obligation ou une preuve significative de l'aptitude du laboratoire à produire des résultats d'essais fiables [21].

\section{Certification des laboratoires}

La participation aux essais d'aptitude professionnelle nationaux et internationaux permettent d'obtenir deux reconnaissances importantes.

- Au niveau national, la Direction Générale de la Santé par arrêté du Secrétariat à la Santé publie régulièrement une liste des laboratoires français ayant reçu la certification de qualification technique pour la mesure de la radioactivité de l'environnement et des denrées destinées à la consommation option mesure des émetteurs bêta-gamma et mesure des émetteurs bêta purs. Ainsi 30 laboratoires ont été certifiés en 1998 [22].

- Au niveau international, le Centre International de Référence pour la Radioactivité de l'Organisation Mondiale de la Santé définit pour chaque pays le souhaitant un à deux laboratoires de référence nationaux.

\section{Accréditation des laboratoires}

En France, l'accréditation COFRAC du laboratoire est un repère de savoir bien-faire dans le domaine considéré. Elle est obtenue à la suite d'un audit qualité qui porte principalement sur le manuel d'assurance de la qualité du laboratoire, qui regroupe les documents où sont définies et décrites les opérations concernant la gestion des échantillons, des équipements, des demandes d'essai, des résultats, etc. Il doit bien évidemment être à jour de toutes les révisions et conforme aux prescriptions du guide ISO/CEI 25 [9].

En ce qui concerne le mesurage de la radioactivité à des niveaux trouvés dans l'environnement, deux programmes d'accréditation COFRAC sont concernés : le programme $\mathrm{N}^{\circ} 135$ « analyse des radionucléides dans l'environnement » et dans une moindre mesure le programme $\mathrm{N}^{\circ}$ 99-4 « Analyse des contaminants chimiques chez les animaux, dans leurs produits et les denrées alimentaires destinées à l'homme ou aux animaux ». Dans le cadre du programme 135, trois niveaux de radioactivité sont répertoriés,

Tableau I. Caractéristiques des essais d'intercomparaisons et d'aptitude organisés aux niveaux national et international auxquels a participé le Laboratoire de Mesure de la Radioactivité de l'Environnement de l'Institut de Protection et de Sûreté nucléaire.

\begin{tabular}{|c|c|c|c|c|c|c|c|c|}
\hline $\begin{array}{l}\text { Type } \\
\text { d'essai }\end{array}$ & Année & $\begin{array}{l}\text { Organisme } \\
\text { participants }\end{array}$ & $\begin{array}{l}\text { Nb de labo. } \\
\text { à mesurer }\end{array}$ & $\begin{array}{c}\text { Nb de radionucléides } \\
\text { échantillon }\end{array}$ & $\begin{array}{c}\text { Code } \\
\text { d'échantillon }\end{array}$ & $\begin{array}{l}\text { Type } \\
\text { du rapport }\end{array}$ & État & Référence \\
\hline Type B & 1987 & AIEA & 21 & 12 & IAEA-GSF & Herbes & Solide & IAEA/AL/018 \\
\hline Type B & 1993 & AIEA & 134 & 22 & IAEA-134 & Mollusque & Solide & IAEA/AL/062 \\
\hline Type B & 1993 & AIEA & 151 & 25 & IAEA-135 & Sédiments & Solide & IAEA/AL/062 \\
\hline Type B & 1994 & AIEA & 159 & 21 & IAEA-300 & Sédiments & Solide & IAEA/AL/064 \\
\hline Type B & 1996 & AIEA & 160 & 27 & IAEA-326-7 & Sols & Solide & IAEA/AL/100P \\
\hline Type B & 1996 & OPRI & 57 & $1\left({ }^{137} \mathrm{Cs}\right)$ & EE 300 & Eau & Liquide & IRC N 44 \\
\hline Type B & 1997 & OPRI & 59 & $3\left({ }^{137} \mathrm{Cs},{ }^{40} \mathrm{~K},{ }^{241} \mathrm{Am}\right)$ & $62 \mathrm{D} 300$ & Ration alimentaire & Solide & IRC N 45 \\
\hline Type B & 1997 & CNEVA & 21 & $1\left({ }^{137} \mathrm{Cs}\right)$ & $96-1$ & Lait en poudre & Solide & CNEVA 0697 \\
\hline Type B & 1997 & CVL & 2 & 33 & NPL/QCY & Eau & Liquide & \\
\hline Type B & 1997 & OPRI & 34 & $2\left({ }^{137} \mathrm{Cs},{ }^{40} \mathrm{~K}\right)$ & $63 \mathrm{SH} 300$ & Eau & Liquide & OPRI 06/97 \\
\hline Type B & 1998 & OMS & 18 & $2\left({ }^{137} \mathrm{Cs},{ }^{40} \mathrm{~K}\right)$ & 63SH300 & Eau & Liquide & IRC N 46 \\
\hline Type B & 1998 & OPRI & en cours & 3 & 64L300 & Lait en poudre & Solide & En cours \\
\hline Type A & 1998 & CETAMA & en cours & 1 & En cours & Sol & Solide & En cours \\
\hline Type B & 1998 & NIST & en cours & 2 & En cours & Mélange bio. & Solide & En cours \\
\hline
\end{tabular}


allant du domaine des traces à ceux caractéristiques de situations accidentelles.

\section{Conclusion}

La contamination potentielle de l'environnement par des matières radioactives entraîne régulièrement des débats sociétaux. Aussi, dans le cadre institutionnel, mais aussi parfois sous la pression du public et des médias et dans un contexte de contentieux, des mesurages contradictoires sont réalisés sur des échantillons de l'environnement, à la demande des autorités de tutelle, Ministères de la santé, de l'environnement et de l'aménagement du territoire et de l'industrie. Il est aisé d'imaginer l'importance de pouvoir identifier et quantifier précisément une source de contamination de l'environnement, de quantifier les apports respectifs de différentes sources, et/ou d'en exclure certaines. Dans ces situations, la qualité et la fiabilité des résultats de mesurage ne doivent pas être mises en doute afin que les discussions restent dans le champ de l'interprétation des résultats et non dans celui de leur contestation. Le mesurage doit donc s'appuyer sur une métrologie reconnue, référencée éventuellement dans des normes. En apportant une réponse technique sans ambiguïté par l'intermédiaire d'un laboratoire d'essai certifié ou accrédité, l'expertise sur l'impact associée aux résultats de radioactivité gagne en crédibilité et répond alors pleinement à l'attente d'analyse des choix que la société doit porter sur les questions abordées.

La reconnaissance de la qualité des résultats fournis par les laboratoires de mesure des radionucléides est aujourd'hui possible grâce à un effort soutenu d'organisation tant au niveau international que national qui a permis de mettre en place des référentiels normatifs sur lesquels peuvent s'appuyer les plans d'assurance de la qualité des laboratoires.

Les procédures de raccordement/traçabilité à des MRP, sous assurance de la qualité, pour les activités les plus élevées peuvent être satisfaites sans difficultés aujourd'hui. Par contre, dans le domaine des traces, nous avons montré la difficulté d'obtenir des étalons adéquats, et fiables (certifiés). L'émergence d'un fournisseur privé étranger et unique spécialisé dans la commercialisation des étalons de types environnementaux mais limités en terme de matrices et de radionucléides, si elle est confirmée, créerait une situation de monopole qui pourrait conduire à des résultats d'essais interlaboratoires certes plus homogènes, mais pas forcément corrects. De plus, des sociétés privées pourront-elles fournir des étalons qui nécessitent des installations de productions onéreuses que seuls des organismes de recherche nationaux peuvent faire fonctionner ? Ainsi, par exemple la production de plutonium 242, nécessaire aux traçages des rendements radiochimiques, est arrêtée en Europe et aux États-Unis et ne pourra être reprise par une société privée. Il y a donc en ce domaine, matière à réflexion pour l'avenir proche.

En ce qui concerne les matériaux de référence secondaires de types environnementaux, l'effort engagé par l'AIEA depuis des années a permis la constitution d'une banque d'échantillons disponibles. Ceux-ci sont bien répandus et sont facilement disponibles auprès des organismes internationaux (AIEA), européen (Geel), nationaux (NIST) et privés (Promochem). Les caractéristiques en terme d'activité des radionucléides de ces MRS sont données comme des valeurs « recommandées ». Une approche pour améliorer la qualification de ces valeurs, en fait pour les « certifier », reste à entreprendre.

Dans le cas particulier des échantillons dits de «blanc» la principale difficulté réside dans la présence de radionucléides d'origine naturelle, communs dans les matériaux environnementaux à différents niveaux de concentrations. Des échantillons d'eau ultra-pure ou de matrices synthétiques de types résines sont alors utilisés comme blancs. Dans d'autres cas, des objets particuliers, géologiquement isolés des contaminations les plus récentes, sont recherchés. En règle générale, les échantillons de blancs sont spécifiques des laboratoires qui les définissent en fonction des matrices à analyser et des activités à mesurer. En dehors des matériels secondaires, qui dans certains cas peuvent être utilisés lorsque l'activité de certains éléments est donnée comme inférieure à une valeur très basse, ils ne peuvent donc être référencés officiellement.

Les données nucléaires continuent à faire l'objet de discussions régulières, cependant pour certains radionucléides, l'incertitude sur certaines données de base comme l'intensité des émissions atteint encore $5 \%$. Des différences sont également observées pour les valeurs proposées des rapports d'embranchement entre les sources officielles documentant les différentes tables de référence des radionucléides. Or, ces tables, qui sont à la base des bibliothèques de traitement des spectres d'acquisition, ne peuvent être retenues suivant leur origine, américaine ou française de leurs auteurs, ou des laboratoires les utilisant. Les besoins de précisions et les différences doivent donc être explicitées et devraient donc faire l'objet d'études supplémentaires, qui relèvent de la physique, pour aboutir à un consensus international. Ces questions apparaissent aujourd'hui comme fondamentales compte tenu de l'amélioration continuelle des performances des techniques et des équipements, ce qui autorise une amélioration de la précision des mesurages en abaissant le rapport signal de l'échantillon sur celui du mouvement propre de l'installation de mesurage.

Les certifications et accréditations obtenues par un laboratoire ne sont pas un acquis définitif, elles sont remises en cause régulièrement tous les deux ans et à chaque modification dans l'organisation ou des moyens humains et matériels du laboratoire. L'accréditation COFRAC du laboratoire est un investissement humain important à travers les participations régulières à des essais d'aptitudes professionnelles (intercomparaisons interlaboratoires), l'élaboration et les révisions du manuel d'assurance qualité, la traçabilité de toutes les opérations réalisées pour mener à bien les essais, la formation d'un personnel qualifié et en nombre suffisant pour assurer des suppléances à tous les postes afin de maintenir le matériel opérationnel en permanence. La pérennité 
de cet ensemble dépend directement de l'effort de maintien en moyens humains qualifiés (recrutement, stage de formation) et de moyens en investissements et fonctionnement, qui s'ils font défaut, conduisent à l'annulation des certifications et accréditations. Le coût financier en est donc important et doit être justifié par l'existence d'une demande substantielle de mesurages. Bien entendu, il faut alors veiller à ce que l'effort consacré aux mesurages de routine permette également les développements métrologiques nécessaires à l'amélioration des méthodes utilisées par les laboratoires.

L'organisation internationale et nationale est bien sûr l'un des garants qui permet aux laboratoires d'essai de relier leurs travaux à des systèmes relevant de la métrologie des rayonnements ionisants. Cependant il ne faut pas oublier que si le mesurage d'activité relative ne pose pas de difficultés majeures, le mesurage d'activité « absolue » reste complexe, et que dans les deux cas ils sont consommateurs de temps et ne peuvent être réalisés que par un personnel qualifié et expérimenté [23].

\section{References}

1. National Radiological Protection Board, rapport technique, 1995, 6(1).

2. Académie des Sciences, Comité des Applications de l'Académie des Sciences, Rapport commun N 5, avril 1996.

3. National Council on Radiation and Measurements, NCRP Report $\mathrm{N}^{\circ} 58,3^{\text {ème }}$ rev. 1994.

4. Laitinen, The Seven Ages of an Analytical Method. Anal. Chem. 1973, 45(14).
5. ISO, Norme $\mathrm{N}^{\circ}$ 9698, ISO, Genève, 1989.

6. ISO, Norme $\mathrm{N}^{\circ}$ 9696, ISO Genève, 1992.

7. ISO, Norme $\mathrm{N}^{\circ}$ 10703, ISO Genève, 1997.

8. Calmet, D.; Bouisset, P; Kerlau, G.; Allenou J. Agence Internationale de l'Énergie Atomique, Proceedings Paris, 24 28 octobre 1994.

9. ISO, Guide ISO/CEI N ${ }^{\circ} 25,1990$.

10. Prichard, E.; Mackay, G. M.; Points, J. The Royal Society of Chemistry.

11. Horwitz, W.; Kamps, L.; Boyer, K. W. J. Assoc. Off. Anal. Chem. 1980, 63,1344.

12. Parkinson, J. A.; Horril, A. D. Nuc. Instr. Meth. Phys. Res., 1984, 223, 598-601.

13. Nir-El, Y. J. Radioanalytical Nucl. Chem. 1998, 227(1-2), 6774.

14. Ibrahim, S. A.; Webb, S. B.; Kattel, A. J. Radioanalytical Nuc. Chem. 1995, 194(1), 213-219.

15. Sima, O.; Dovlete, C. Appl. Radiat. Isot. 1997, 48(1), 59-69.

16. OECD Environmental Monograph $\mathrm{N}^{\circ}$ 45, OECD, Paris, 1992.

17. Simoen, J. P.; Amoury, F. Bull. BNM 1995, 100, 15-34.

18. Promochem. Reference material for macro-, micro- and trace element analysis catalogue.

19. Agence internationale de l'énergie atomique, Catalogue for Reference Materials and intercomparison. Exercises 1998/1999, Analytical Quality Control Services, 1998.

20. Fournier, M.; Calmet, D. ESARDA Bull., EUR 17312 EN, 1997, 233-236.

21. ISO, Guide ISO $N^{\circ} 2$, ISO Genève.

22. Rep. Française, Journal Officiel, Arrêté du 22 mai 1998.

23. Debertin, K. Appl. Radiat. Isot. 1996, 47(4), 423-431. 